\title{
Influences of Movement Behavior on Animal Distributions at Edges of Homogeneous Patches
}

\author{
Hilary C. Young, ${ }^{1}$ Tyler G. Reid, ${ }^{2}$ Lea A. Randall, ${ }^{3}$ Leanna E. Lachowsky, \\ Danusha J. Foster, ${ }^{4}$ Chris J. Pengelly, ${ }^{5}$ Tanya Latty, ${ }^{6}$ and Mary L. Reid ${ }^{1}$ \\ ${ }^{1}$ Department of Biological Sciences, University of Calgary, 2500 University Drive NW, Calgary, AB, Canada T2N 1N4 \\ ${ }^{2}$ Department of Transmission and Distribution, SNC-Lavalin Environment, 4th Floor, 9095 th Avenue SW, \\ Calgary, AB, Canada T2P $3 G 5$ \\ ${ }^{3}$ Conservation Research Centre, Calgary Zoo, 1300 Zoo Road NE, Calgary, AB, Canada T2E 7V6 \\ ${ }^{4}$ Faculty of Medicine, University of Toronto, 1 Kings College Circle, Toronto, ON, Canada M5S 1A8 \\ ${ }^{5}$ Department of Environmental Services, Stantec Incorporated, 300-675 Cochrane Drive, Markham, ON, Canada L3R OB8 \\ ${ }^{6}$ School of Biological Sciences A12, University of Sydney, Sydney, NSW 2006, Australia \\ Correspondence should be addressed to Hilary C. Young; hcyoung@gmail.com
}

Received 24 July 2012; Revised 24 December 2012; Accepted 28 December 2012

Academic Editor: Mary C. Christman

Copyright (C) 2013 Hilary C. Young et al. This is an open access article distributed under the Creative Commons Attribution License, which permits unrestricted use, distribution, and reproduction in any medium, provided the original work is properly cited.

\begin{abstract}
We propose that changes in movement behavior may be a proximate mechanism that influences the accumulation of animals at habitat edges. We tested this idea with a combination of empirical and simulation experiments in a resource-free landscape. The movements of individual flour beetles, Tribolium confusum, were tracked across a paper arena edged with invisible tape until beetles crossed the edge. Movement behavior (step lengths and turn angles) and cumulative occupancy were analyzed according to distance from the edge. We found that beetles took smaller steps with larger turn angles near edges than in the center of the arena and that beetle distribution was highly biased towards the edge of the arena. We then tested two agent-based simulation models for each beetle: an edge-independent model and an edge-dependent model. Both models predicted less time spent at the edge than was observed. The proportion of time spent at edges depended on the propensity to cross the edge, which could not be explained by beetle body size or energetic condition. The distribution of animals with respect to habitat edges depends on many factors, but we suggest that proximate mechanisms such as movement behavior should be explicitly considered when interpreting animal distributions.
\end{abstract}

\section{Introduction}

Movement of individuals is a critical factor for the population ecology of most organisms, affecting energy flow, distribution, and the genetic and demographic structure of populations [1-4]. Knowledge of the causes of movement behavior may be key in developing a full understanding of the spatial structure and dynamics of populations [1, 5-10]. For example, an increasingly large body of research finds that organisms generally move quickly and directly through low-quality habitats (e.g., $[4,11,12])$. This in turn predicts there will be fewer individuals in resource-poor than in resource-rich environments $[5,13]$, and many studies have found such a correspondence between movement behavior and population density (e.g., [14-18]).

In addition to habitat quality, another key factor affecting the movement of organisms is the degree of permeability of the interface between habitats [16, 19-21]. Permeability is the tendency of the edge to inhibit or enhance organisms' movement across it $[19,21-23]$ and it directly affects the degree to which organisms leave a particular habitat $[12,15$, 19]. Borders that are readily crossed by dispersing individuals are considered to be soft or semipermeable edges, while those that are unlikely to be crossed are known as hard or impermeable. This distinction may be a result of the environment (a physical barrier, for instance), or a behavioral 
response from the animal itself $[12,16,19,24]$. Behavioral responses may vary inter- and intraspecifically according to species' traits (e.g., specificity of diet, migratory status, and scale of movement) and according to the characteristics of individuals (e.g., age, sex, reproductive state, and condition). Individuals' movement patterns at edges may also vary as a result of personality: the propensity of animals to explore or take on risks can be governed by whether or not they are innately bold [25, 26], aggressive, active, or social [27].

While the notions of habitat-dependent movement behavior and edge permeability are each well established, links between them remain unclear although long recognized as of interest [28]. We suggest they may share common mechanistic determinants of movement. A widely applied way of assessing changes in movement behavior is to divide a movement path into a series of step lengths and turn angles [29]. In this model, longer step lengths and a narrower distribution of turn angles characterize directed movement, whereas shorter step lengths and a wider range of turn angles typify more random movement. As noted above, directed movement can cause animals to quickly leave habitats with few resources, while more random movement behavior results in longer durations in an area. If encounters with an edge cause individuals to shorten their step lengths or increase their turn angles, then we should see an increased density of individuals at the edge. Previously, high population density at edges has been attributed to the presence of complementary or supplementary resources in two adjacent habitats, or to higher habitat quality at the edge itself (e.g., $[30,31])$. We suggest that the pattern could arise from altered movement behavior at edges regardless of resource distribution.

The idea that movement behavior alone could explain that accumulation at edges has been proposed occasionally (e.g., [32, 33]), but to our knowledge there have been no empirical tests within patches where resources are known to be homogeneous. Some studies have found evidence of accumulation at edges without an associated increase in resources, but have not investigated the mechanism behind the accumulation. For example, Olofsson et al. [34] found that reindeer grazed vegetation more intensely within $50 \mathrm{~m}$ of a fence placed across continuous habitat than they grazed further away. Because there is no obvious change in intrinsic habitat quality with distance to the fence, this observation could be consistent with animals accumulating near the fence because of their movement behavior. However, this movement-based hypothesis has not been explicitly tested.

To examine how movement behavior at edges might explain distribution at edges, we analyzed the movement paths of the confused flour beetle, Tribolium confusum Jacquelin du Val (Tenebrionidae) in a resource-free arena (or "patch") bounded by a distinct but crossable edge. Tenebrionid beetles have previously been used to investigate animal responses to landscape patchiness because of their small size and propensity to move in only two dimensions (e.g., $[3,7,35])$. Furthermore, a recent study of edge responses in beetles found that nearly $60 \%$ of the 78 most common species encountered in New Zealand exhibited a significant change in abundance within two meters of the edge [36], which makes them an appropriate taxon for edge studies at small scales. We asked three main questions. First, are individuals equally likely to be in any part of the patch, or is their distribution biased with respect to the edge? Second, do movement behaviors, measured as step length and turn angles, differ between the edge and the center of the patch? And third, can beetle distribution in a bounded patch be explained simply in terms of step lengths and turn angles, or might additional mechanisms such as edge permeability or individual differences in behavior play a role?

The use of simple models in studies of movement ecology can facilitate the generalization of findings across taxa and habitats [37], but they are rarely tested with empirical data [38]. To answer the questions we posed above, we first observed the distribution and movement behaviors of individual beetles with respect to their distance from the edge of the experimental arena. We then developed an individualbased computer simulation that mimicked the conditions of the empirical experiment. The model drew step lengths and turn angles from the distributions that resulted from the experiment and simulated beetle movement using a correlated random walk. We predicted that if step length and turn angles determine beetle distribution with respect to the edge, we would be able to recreate the distributions observed in the empirical experiments in the simulation models.

\section{Methods}

2.1. Empirical Study. Tribolium confusum (3-5 mm in length, $0.5-1 \mathrm{~mm}$ in width) were obtained from a laboratory population that has been reared for fifteen years at the University of Calgary. Beetles were housed in a container of dry white wheat flour at room temperature. The experimental arena consisted of a $20 \mathrm{~cm} \times 20 \mathrm{~cm}$ sheet of paper attached to a board on all four sides with $19 \mathrm{~mm}$ wide Scotch Magic tape (a semipermeable "edge" that obstructed but did not prevent movement across it). The tape differed in texture from the paper, and although it provided no vertical barrier, Morales and Ellner [35] have shown that T. confusum are unlikely to cross such tape. The arena was marked into seven concentric zones of $1.5 \mathrm{~cm}$ in width, which were numbered from the center outwards (Figure 1). The outer zone (zone 7a) also contained corner zones, each of which was $2.25 \mathrm{~cm}^{2}$ in area. These four corner areas were collectively known as zone $7 \mathrm{~b}$. Our zones allowed us to quantify time spent near and far from the edge of the arena in such a way that could be compared to a simulation model.

We conducted the experiment in a darkroom with a single incandescent light $(60 \mathrm{~W})$ placed $60 \mathrm{~cm}$ above the arena to evenly illuminate it. The light was turned on $15 \mathrm{~min}$ prior to the experiment to allow the temperature to stabilize; the mean temperature of trials was $25.8 \pm 0.09^{\circ} \mathrm{C}$. One beetle was observed at a time, and a new experimental arena was used (Figure 2) for each trial to eliminate the potential confounding effect of residual chemical cues from previous beetles [39]. Before each trial began, the test beetle was placed in the center of the arena covered by a transparent plastic vial $(2.5 \mathrm{~cm}$ diameter $)$ for one minute to reduce 


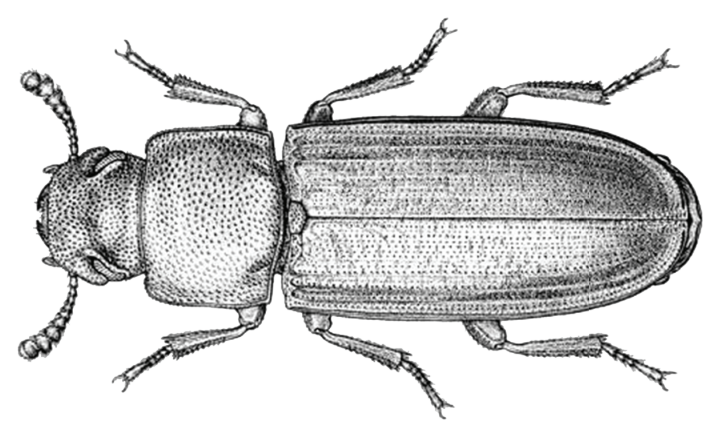

FIGURE 1: Drawing of adult Tribolium confusum (reprinted with permission from Canada Grains Commission, http://www.grainscanada.gc.ca/storage-entrepose/pip-irp/cfb-tbf-eng.htm). Body length ranges from 3 to $5 \mathrm{~mm}$.

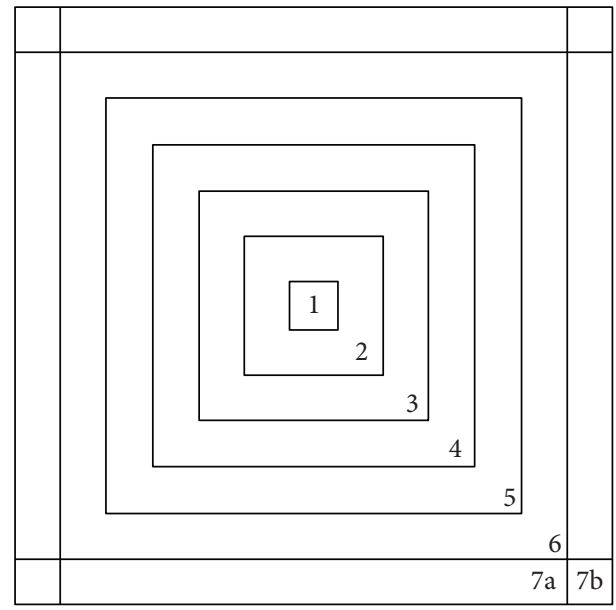

Figure 2: The $20 \mathrm{~cm} \times 20 \mathrm{~cm}$ experimental arena was divided into 7 concentric zones. Width of each zone was $1.5 \mathrm{~cm}$. The outer zone (zone $7 \mathrm{a}$ ) was interrupted at each corner by a $2.25 \mathrm{~cm}^{2}$ zone (zone $7 b)$.

agitation dispersal $[40,41]$. The vial encompassed all of zone 1 and extended into zone 2; therefore, for the purposes of analysis, zone 1 was incorporated into zone 2. Each trial was recorded using a digital camera (DCR-TRV 230, Sony Ltd.) (60 frames $\mathrm{sec}^{-1}$ ) fixed directly above the arena. The trial began immediately after the one-minute acclimation period and ended after the beetle had left the experimental arena $(n=42)$ or after 600 seconds $(n=12)$, whichever occurred first. At the end of each trial, the beetle under observation was weighed to the nearest $0.1 \mathrm{mg}$ and its length and width measured with a dissecting scope fitted with an ocular micrometer at $10 \mathrm{X}$ magnification. Beetle condition index was calculated as the residual of the regression of mass on volume (estimated as a cylinder) [42].

To determine the distribution of time spent in each zone from the digital videos of the trials, we used an event recorder (Jwatcher 1.0: http://www.jwatcher.ucla.edu/). Time spent in each zone was summed for each individual. We recorded edge encounter events as the extension of the beetle's head past the interior edge of the transparent tape.
To map the paths of beetles, we used motion analysis software (Tracker 1.7.2: http://www.cabrillo.edu/ dbrown/tracker/) to determine each beetle's coordinates at 1-second intervals from which step lengths and turn angles were calculated. Each turn angle was recorded as the angle of change in direction from the previous step. These angles were bounded between 0 and $180^{\circ}$. We analyzed the first and last 30 seconds of each trial but found little difference in step lengths and turn angles between the two time periods. We therefore pooled data from both periods in our analyses. We used JMP 9.0 (SAS Inc. statistical software) and Oriana 2 (http://www.kovcomp.co.uk/oriana/newver2.html) to analyze the distribution of step lengths, and turn angles respectively.

2.2. Simulation Models. We modeled each beetle's movement using agent-based software (Netlogo 3.1.3: http://ccl.northwestern.edu/netlogo/). The conditions of the empirical experiment were replicated as closely as possible-beetles in the model began the trial in the center of an arena with the same dimensions and zones as the experimental arena. We modeled the movement of each individual beetle in our empirical trial as a correlated random walk $[29,43]$. Beetles in our simulation model took a step each second, and the length of the step and turn angle relative to the previous step were chosen from the distribution of observed movement behaviors of each particular beetle. Therefore, distribution parameters were unique to each beetle. The observed distributions of step lengths and turn angles were consistent with a normal distribution when square-root and ln-transformed, respectively. Values were randomly drawn from the transformed distributions and then back-transformed; these angles were then applied relative to the beetle's previous turn angle to move the beetle its next step.

As with the empirical trials, simulated trials ended either after a beetle had left the arena or 600 time steps (seconds) had elapsed. We initially ran two versions of the model. The edge-independent model assumed that beetles did not alter their movement behavior based on their location within the arena. For this model, distributions were determined for the step lengths and turn angles of each beetle over all zones. The edge-dependent model included different movement behaviors for the center zones (zones 2-6), the edge zone (zone $7 \mathrm{a}$ ), and the corner zone (zone $7 \mathrm{~b}$ ) according to the observed distributions of step lengths and turn angles in these zones for each beetle. We combined center zones because we observed little difference in the movement behavior of beetles among these zones (analyses not shown). Each version of the model was replicated 50 times for each of the 54 beetles. The output of each replicate was the number of seconds the simulated beetle was in each of the 7 zones.

2.3. Data Analysis. We employed an individual-based analysis to examine the movement behavior of beetles in our empirical trials and in our models. We calculated the time spent in each zone for each beetle in the experimental study and for both models and transformed $(\log (x+0.1)$, where 
$x=$ summed time) these values meet the assumptions of parametric tests, including normality, homogeneity of variance, and independent errors. The proportion of time individual beetles spent in each zone was then compared to the proportion of time expected based on the area of that zone (calculated as trial length/zone area) with a onesample $t$-test. We used simple linear regression to consider the effect of individual beetle and zone of the arena (center, edge, and corner) on ln-transformed step lengths and turn angles. We compared the cumulative time spent at the edge zone in the empirical study and in the models using mixed linear models with individual beetle as a random effect. Finally, to address the influence of additional factors on beetle distribution across the experimental arena, we used multiple linear regression to determine whether beetle traits (body volume, and condition index), beetle movement behavior, and the response to edge (crosses per encounter) explained the proportion of time an individual spent at the edge. Statistical significance of regression analyses was determined via $F$-tests. For all analyses, we used a significance value of $P<0.05$ as the criterion for rejecting the null hypothesis.

\section{Results}

\subsection{Empirical Study}

3.1.1. Distribution within the Arena. Observed trial lengths varied from 8.4 to the imposed maximum of $600 \mathrm{sec}$ (median $=47.0 \mathrm{sec}, n=54$ ). The time beetles spent in each zone was not proportional to the area of each zone (Figure 3(a)). Beetles spent significantly more time than expected at the edge (zone $7 \mathrm{a}: t_{52}=4.90, P<0.0001$ ) and corners (zone $7 \mathrm{~b}: t_{52}=6.15, P<0.0001$ ). Conversely, they spent less time than expected in the middle zones (zone 4: $t_{52}=-8.69$, zone 5: $t_{52}=-17.37$, and zone $6: t_{52}=$ -11.25 ; all: $P<0.0001)$. Time spent in the innermost zones was proportional to those areas of the arena (zone 2: $t_{52}=$ 1.39, $P=0.17$, zone $\left.3: t_{52}=-2.00, P=0.051\right)$.

3.1.2. Movement Behavior and Distribution at Edges. Beetles took smaller steps (Figure 4(a)) with larger turn angles (Figure $4(b)$ ) in the edge zone (7a) than in the center zones (zones 2-6). The mean difference in step length (95\% CI) was $1.36 \mathrm{~mm}(1.25-1.49 \mathrm{~mm}$, back-transformed from analysis of ln-transformed data; $t_{48}=7.08, P<0.0001$ ), while turn angles decreased by $11.9^{\circ}$ on average (95\% CI: $5.6-18.2^{\circ}$; $\left.t_{48}=-3.78, P<0.0005\right)$. Individual differences were evident in that mean step lengths at the edge were positively correlated with mean step lengths in the center (Figure 4(a); $r_{48}=0.85, P<0.0001$ ), but there was no consistency within individuals in the relative magnitude of turn angles at the center and edge (Figure $4(\mathrm{~b}) ; r_{48}=0.12, P>0.4$ ).

3.2. Simulation Models. The results of our two simulation models, with edge-independent or edge-dependent behavior, differed from each other but neither replicated the distribution of time spent in each zone by beetles in our experimental study (Figures 3(b) and 3(c) versus 3(a)). The edge-dependent

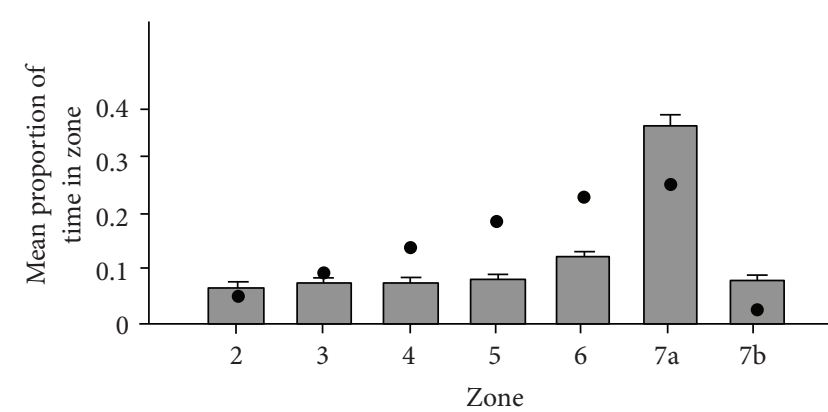

(a)

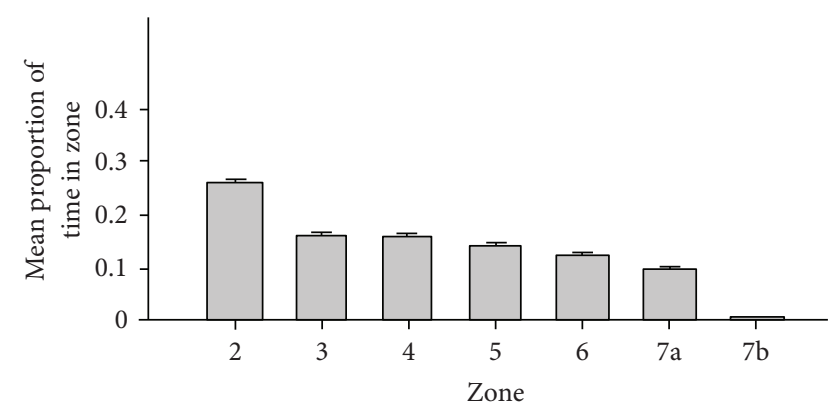

(b)

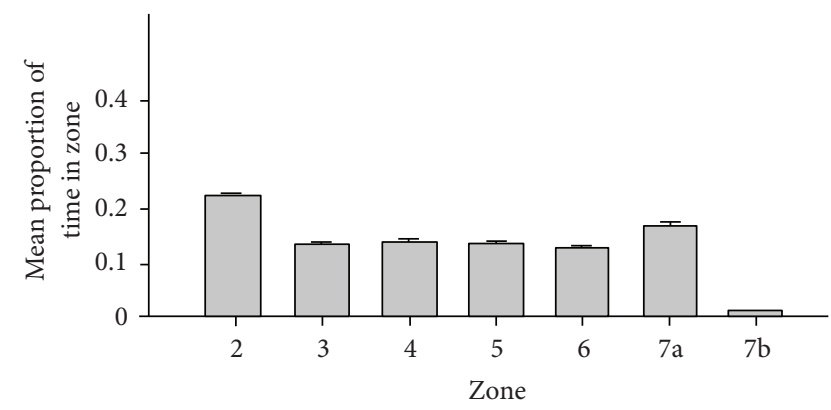

(c)

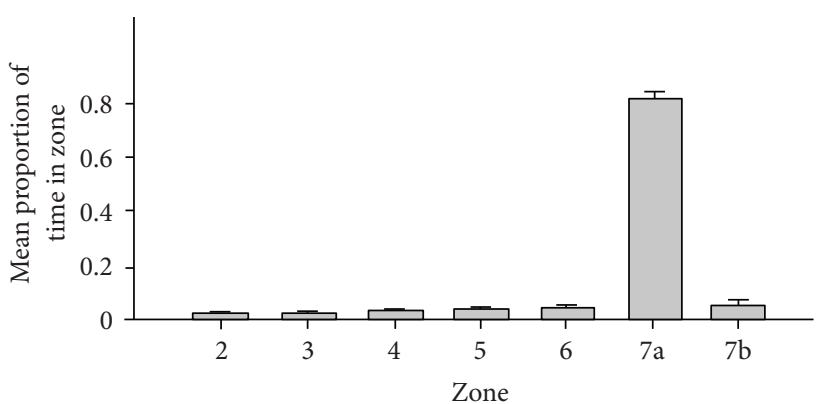

(d)

FIGURE 3: The mean proportion, +/-SE, of time Tribolium confusum beetles ( $n=54)$ spent in each zone in (a) the empirical study, (b) the edge-independent (EI) simulation model, (c) the edge-dependent (ED) simulation model, and (d) the ED model using the maximum difference in behavior between the center and edge zones. Black dots indicate the proportion of the arena for each zone. 


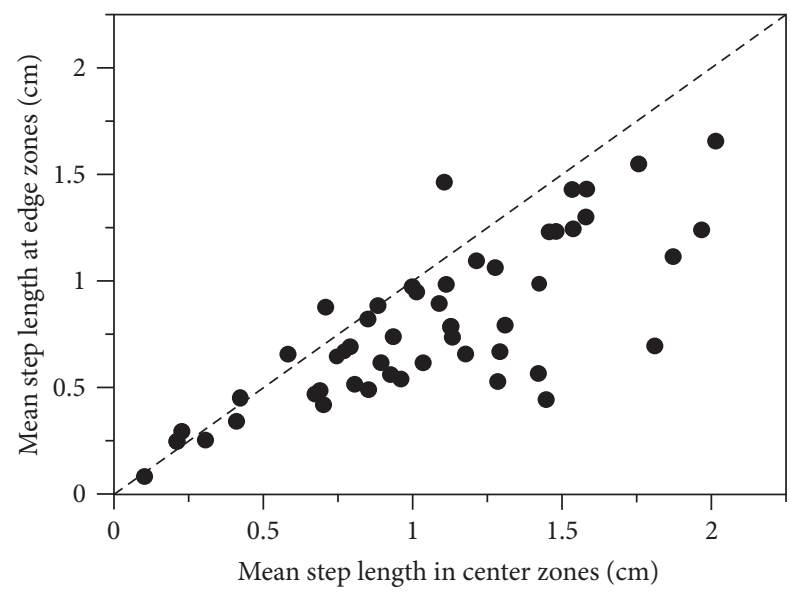

(a)

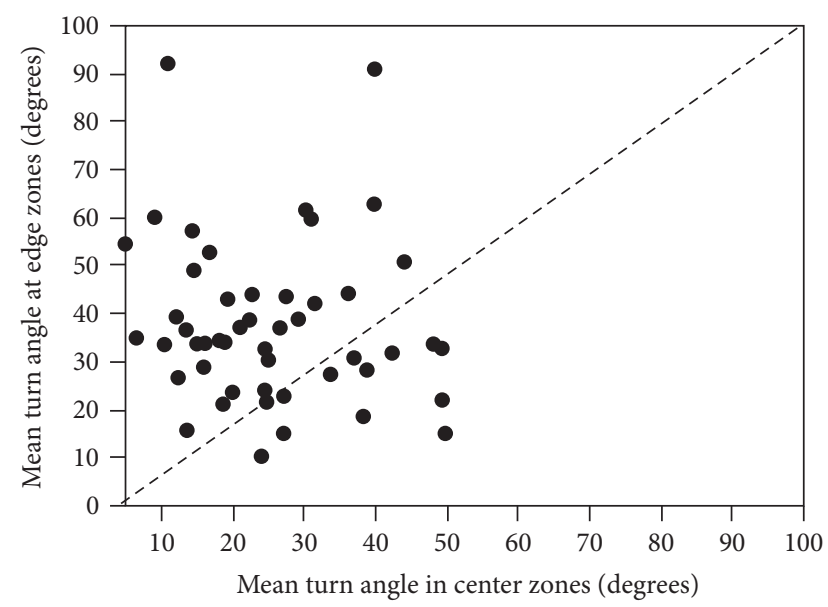

(b)

FIGURE 4: Movement behavior of individual beetles at the edge (zone 7a) relative to their movement in center zones (zones 2-6) for (a) mean step lengths for beetles that took more than one step in each zone area and (b) mean turning angles for beetles that made more than one turn in each zone area. Dashed line indicates equal values at the center and edge.

model resulted in a higher proportion of time being spent at the edge zone (zone 7a) than the edge-independent model $\left(F_{1,96}=15.56, P=0.0002\right)$. Conversely, less time was spent in the innermost zones (zones 2,3) and in zone 4 in the edge-dependent model than in the edge-independent model. However, despite differences in magnitude, both models predicted less time spent near the edge (in zone $7 \mathrm{a}$ ) and more time spent near the center (zones 2 through 6) than was actually observed (Figure 3).

Given the above results, we remodeled beetle movement using only the step lengths and turns angles that were most different between the center and the edge observed for any beetle in the empirical trials. We wanted to explore whether any changes in movement behavior between center and edge, within the range of those observed, would result in a distribution of beetles' use of the arena that was similar to our empirical results. As expected from our original
TABLE 1: Model prediction of the proportion of time spent in the edge zone. $R^{2}=0.50, n=45$.

\begin{tabular}{lcccc}
\hline \multirow{2}{*}{ Variable } & \multicolumn{4}{c}{ Parameter } \\
& Estimate & SE & $t$ & $P$ \\
\hline $\begin{array}{l}\text { Intercept } \\
\begin{array}{l}\text { Mean center step length } \\
\text { (mm) }\end{array}\end{array} 0^{0.40}$ & 0.045 & 8.86 & $<0.0001$ \\
$\begin{array}{l}\text { Change in mean step length } \\
\text { (center-edge; mm) }\end{array}$ & -0.049 & 0.055 & 2.39 & $<0.03$ \\
$\begin{array}{l}\text { Mean center turn angle } \\
\text { (rad) }\end{array}$ & -0.003 & 0.002 & -0.73 & 0.47 \\
$\begin{array}{l}\text { Change in mean turn angle } \\
\text { (center-edge; rad) }\end{array}$ & -0.0004 & 0.002 & -0.26 & $>0.057$ \\
\begin{tabular}{l} 
Crosses per encounter \\
\hline
\end{tabular} & -0.37 & 0.064 & -5.76 & $<0.0001$ \\
\hline
\end{tabular}

hypothesis, the short steps and large turn angles at the edges, relative to the center, resulted in beetles spending most of their time near the edge (Figure 3(d)). However, this modification to the edge-dependent simulation model did not succeed in replicating the distribution of beetles in our empirical experiment. This version of the model produced a distribution in which twice as much time was spent in the edge zones than beetles had spent in the empirical study $(80 \%$ versus $40 \%$ ) (Figure 3(d)).

To address the influence of additional factors on beetle distribution across the experimental arena, we investigated whether beetle traits, beetle movement behavior, and the response to edge (crosses per encounter) explained the proportion of time an individual spent at the edge. Beetle volume and condition did not significantly affect time at the edge (both $P>0.5$ ) and were removed from subsequent models. Some movement behaviors had small but significant effects on the proportion of time at the edge. Beetles that took larger steps with smaller turn angles at the center tended to spend more time at the edge (Table 1). However, their behavior near edges did not detectably affect the time they spent there, whether measured as the change in behavior at the edge (Table 1) or when only edge step lengths and turn angles were used in models ( $P>0.6$, analyses not shown). The strongest predictor of the proportion of time spent at the edge was the proportion of encounters with an edge that resulted in the beetle crossing an edge (Table 1). When the probability of crossing upon encountering an edge was low, the proportion of time spent at the edge was higher (Figure 5). Furthermore, the ratio of crosses per encounter was affected neither by body volume $\left(F_{1,49}=1.15, P=0.29\right)$ nor by condition index $\left(F_{1,49}=2.64, P=0.11\right)$.

\section{Discussion}

The beetles in our experiment were surrogates for any organism capable of movement. Our experimental microlandscape was resource-free and, from the beetles' point-of-view, was comprised of two habitat elements: the paper arena and the taped edge. For beetles born and raised in a container of flour, the arena was likely to seem foreign and exposed. 


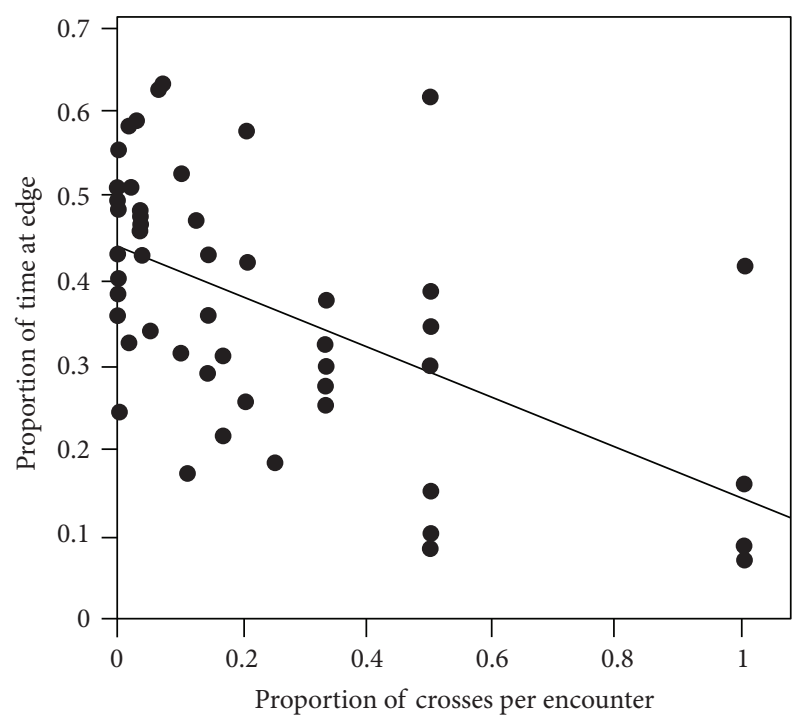

FIGURE 5: The proportion of crosses per encounter with the edge as a function of time spent in the edge zone during the empirical trials. Edge encounters were defined as the number of times a beetle extended its head beyond the inner edge of the tape surrounding the arena. Because trials ended once a beetle crossed the edge (i.e. exited the arena), the proportion of crosses per encounter is always $1 / x$, where $x$ is the number of edge encounters.

It is reasonable to assume that beetles were motivated to find a better habitat (see [35]) but there was no reason to think that they should persist in trying to go in any particular direction. Thus our null hypothesis was that beetles would use the whole arena equally such that the time they spend in a given zone at some distance from the edge should be proportional to the size of that zone. The taped edge had virtually no vertical dimension and was likely not detectable from any distance. However, beetles encountering this slippery surface were unlikely to cross it to leave the experimental arena. This experimental design allowed us to consider the effect of a semipermeable boundary on the movement patterns and resulting distribution of organisms in a controlled environment on a small, manageable scale.

We found that beetles spent more time at edges and corners and less time in interior zones than expected from the area of each zone (Figure 3(a)). This occurred despite the fact that beetles were initially placed in the center of the arena. Campbell and Hagstrum [44] obtained similar results for the congeneric beetle $T$. castaneum, in that they found that beetles tended to be located near the edges of food patches. As in our study, they found this was due in part to beetles traveling more slowly along edges than farther away from them. The observation that animals often accumulate near edges is not new ([32, 45], and references therein), and indeed certain bird and mammal species are commonly considered to be "edge species" (but see [31, 46, 47]) for critical discussion of this term]. The underlying reasons for these distribution patterns often remain unclear because of the sheer number of variables that must be taken into consideration in natural systems. Our system suggests that an edge alone, without any evident edge-associated changes in resources, can result in greater animal activity at edges.

Our study proposed the hypothesis that disproportionate activity at edges could be explained mechanistically by changes in step lengths and turn angles. We did observe the proposed mechanism, namely, shorter step lengths and higher turn angles at edges than at the center and disproportionate time spent at edges in our putatively resource-free environment. Qualitatively, these movement patterns predict increased time spent at edges, as we observed. Quantitatively, however, we did not recreate the observed distributions using mean movement behaviors in centers and at edges in two simulation models (Figures 3(b) and 3(c)) or using "extreme" movement behaviors in a third (Figure 3(d)). Thus while it is possible that a combination of turn angles and step lengths could be found that would ultimately predict the observed distributions, it seems more likely that that there are more complex edge behaviors involved. It is important to note, however, that although the mechanism tested was not sufficient to explain the distribution of beetles with respect to edges in our study, it is a process that should be considered when integrating multiple paradigms to explain animal movement [10].

Generally, beetles encountered the edge a number of times before they crossed (Figure 5). It seems plausible that there was something fundamentally different about their behavior at the edge that we did not capture with our simple model of step lengths and turn angles. This behavioral change may be related to the beetles' perceptual range, as they appeared not to perceive the edge until they were within millimeters of it. Many studies of animal movement have found evidence of a "boundary effect" wherein organisms perceive and respond to both semipermeable and impermeable habitat edges (e.g., [1, 4, 14, 16, 48, 49]) but few studies have clearly linked edge behavior to distribution with respect to the edge.

Crossing the boundary may be a result not of movement ability so much as movement propensity, or the probability that an individual will cross a boundary once it reaches an edge [50]. Habitat edges acting as impermeable or semipermeable boundaries may significantly affect the trajectory of movement paths in a number of species (e.g., $[1,49]$ ). Indeed, there is increasing evidence that animals change their movement patterns upon encountering edges (e.g., [15, 5153]). Correlated random walks may not describe movement at edges well, for example, if animals "reflect" off boundaries and take a new direction at random, if animals respond to edges by linearizing their movement paths parallel to them, or if animals otherwise shift movement modes at edges (i.e., from a correlated random walk to a biased correlated random walk, sensu Crone and Schultz [54]). Future work incorporating explicit behavioral responses at edges into individual-based models could provide more insight into how animals are distributed around edges.

Despite beetles' general propensity to avoid crossing the edge upon their first encounter, they differed in the number of times they encountered the tape before passing over it and out of the arena (Figure 5). We interpret this to mean that some beetles considered the tape to be a hard edge, while other beetles did not perceive the tape as an edge at all. It is 
possible that individual beetles responded differently to edges due to factors such as sex, reproductive state, condition, and personality (i.e., boldness). We found that the number of edge encounters prior to leaving was significantly related to the time beetles spent in the edge zone (Table 1). However, we did not find any correlation between beetle condition or body size and time spent in the edge zone.

The beetles in this study exhibited a considerable amount of variation not just in their likelihood to cross the edge, but also in their movement behaviors (Figure 4). However, despite this variation, individual beetles were generally consistent within themselves in that if they took large steps in the center, they took large steps at edges (Figure 4). These results support the notion that population processes may depend more on the behaviors of a few bold individuals than on an average individual [55].

Our empirical study found that animal activity can be concentrated at edges even without a change of resources there. Thus, animal distribution at edges may not always reflect resources (cf. [30]) or as a consequence, follow an ideal free distribution. Rather, the accumulation of individuals at edges may influence resources. For example, one of the consequences of higher reindeer activity near fences is altered ecosystem processes [34]. Aggregation of individuals due to movement behavior at edges may also influence community structure [33]. Understanding the complex behavior of animals at edges independent of resources will help clarify population processes, which is critical as habitats become increasingly fragmented.

\section{Acknowledgments}

The authors thank Abbie Stewart and Josée Méthot for technical assistance and Petr Komers and Elizabeth Crone for their helpful and constructive comments on this work. This work was supported by NSERC scholarships to HCY, LEL and TL, a NSERC Discovery Grant to MLR, and the University of Calgary.

\section{References}

[1] A. R. Johnson, J. A. Wiens, B. T. Milne, and T. O. Crist, "Animal movements and population dynamics in heterogeneous landscapes," Landscape Ecology, vol. 7, no. 1, pp. 63-75, 1992.

[2] N. Shigesada and K. Kawasaki, Biological Invasions: Theory and Practice, Oxford Series in Ecology and Evolution, Oxford University Press, Oxford, UK, 1997.

[3] N. E. Mcintyre and J. A. Wiens, "How does habitat patch size affect animal movement? An experiment with darkling beetles," Ecology, vol. 80, no. 7, pp. 2261-2270, 1999.

[4] N. Schtickzelle, A. Joiris, H. van Dyck, and M. Baguette, "Quantitative analysis of changes in movement behaviour within and outside habitat in a specialist butterfly," BMC Evolutionary Biology, vol. 7, article 4, 2007.

[5] P. Turchin, "Translating foraging movements in heterogeneous environments into the spatial distribution of foragers," Ecology, vol. 72, no. 4, pp. 1253-1266, 1991.
[6] J. A. Wiens, N. C. Stenseth, B. van Horne, and R. A. Ims, "Ecological mechanisms and landscape ecology," Oikos, vol. 66, no. 3, pp. 369-380, 1993.

[7] T. O. Crist and J. A. Wiens, "Individual movements and estimation of population size in darkling beetles (Coleoptera: Tenebrionidae)," Journal of Animal Ecology, vol. 64, no. 6, pp. 733-746, 1995.

[8] P. Stapp and B. van Horne, "Response of deer mice (Peromyscus maniculatus) to shrubs in shortgrass prairie: linking smallscale movements and the spatial distribution of individuals," Functional Ecology, vol. 11, no. 5, pp. 644-651, 1997.

[9] D. E. Bowler and T. G. Benton, "Causes and consequences of animal dispersal strategies: relating individual behaviour to spatial dynamics," Biological Reviews of the Cambridge Philosophical Society, vol. 80, no. 2, pp. 205-225, 2005.

[10] R. Nathan, W. M. Getz, E. Revilla et al., "A movement ecology paradigm for unifying organismal movement research," Proceedings of the National Academy of Sciences of the United States of America, vol. 105, no. 49, pp. 19052-19059, 2008.

[11] T. Crist, D. Guertin, J. Wiens, and B. Milne, "Animal movement in heterogeneous landscapes: an experiment with Eleodes beetles in shortgrass prairie," Functional Ecology, vol. 6, no. 5, pp. 536-544, 1992.

[12] A. Berggren, B. Birath, and O. Kindvall, "Effect of corridors and habitat edges on dispersal behavior, movement rates, and movement angles in Roesel's bush-cricket (Metrioptera roeseli)," Conservation Biology, vol. 16, no. 6, pp. 1562-1569, 2002.

[13] D. Kuefler, B. Hudgens, N. M. Haddad, W. F. Morris, and N. Thurgate, "The conflicting role of matrix habitats as conduits and barriers for dispersal," Ecology, vol. 91, no. 4, pp. 944-950, 2010 .

[14] C. B. Schultz, "Dispersal behavior and its implications for reserve design in a rare Oregon butterfly," Conservation Biology, vol. 12, no. 2, pp. 284-292, 1998.

[15] N. M. Haddad, "Corridor use predicted from behaviors at habitat boundaries," American Naturalist, vol. 153, no. 2, pp. 215-227, 1999.

[16] C. B. Schultz and E. E. Crone, "Edge-mediated dispersal behavior in a prairie butterfly," Ecology, vol. 82, no. 7, pp. 1879-1892, 2001

[17] B. G. Dickson, J. S. Jenness, and P. Beier, "Influence of vegetation, topography, and roads on cougar movement in Southern California," Journal of Wildlife Management, vol. 69, no. 1, pp. 264-276, 2005.

[18] D. Kuefler and N. M. Haddad, "Local versus landscape determinants of butterfly movement behaviors," Ecography, vol. 29, no. 4, pp. 549-560, 2006.

[19] J. A. Stamps, M. Buechner, and V. V. Krishnan, "The effects of edge permeability and habitat geometry on emigration from patches of habitat," American Naturalist, vol. 129, no. 4, pp. 533-552, 1987.

[20] W. F. Fagan, R. S. Cantrell, and C. Cosner, "How habitat edges change species interactions," American Naturalist, vol. 153, no. 2, pp. 165-182, 1999.

[21] W. Z. Lidicker, "Responses of mammals to habitat edges: an overview," Landscape Ecology, vol. 14, no. 4, pp. 333-343, 1999.

[22] M. Buechner, "Conservation in insular parks: simulation models of factors affecting the movement of animals across park 
boundaries," Biological Conservation, vol. 41, no. 1, pp. 57-76, 1987.

[23] P. Duelli, M. Studer, I. Marchand, and S. Jakob, "Population movements of arthropods between natural and cultivated areas," Biological Conservation, vol. 54, no. 3, pp. 193-207, 1990.

[24] O. Kindvall, "Dispersal in a metapopulation of the bush cricket, Metrioptera bicolor (Orthoptera: Tettigoniidae)," Journal of Animal Ecology, vol. 68, no. 1, pp. 172-185, 1999.

[25] D. S. Wilson, A. B. Clark, K. Coleman, and T. Dearstyne, "Shyness and boldness in humans and other animals," Trends in Ecology and Evolution, vol. 9, no. 11, pp. 442-446, 1994.

[26] M. Wolf, G. S. van Doorn, O. Leimar, and F. J. Weissing, "Lifehistory trade-offs favour the evolution of animal personalities," Nature, vol. 447, no. 7144, pp. 581-584, 2007.

[27] D. Réale, S. M. Reader, D. Sol, P. T. McDougall, and N. J. Dingemanse, "Integrating animal temperament within ecology and evolution," Biological Reviews, vol. 82, no. 2, pp. 291-318, 2007.

[28] S. L. Lima and P. A. Zollner, "Towards a behavioral ecology of ecological landscapes," Trends in Ecology and Evolution, vol. 11, no. 3, pp. 131-135, 1996.

[29] P. M. Kareiva and N. Shigesada, "Analyzing insect movement as a correlated random walk," Oecologia, vol. 56, no. 2-3, pp. 234-238, 1983.

[30] L. Ries and T. D. Sisk, "A predictive model of edge effects," Ecology, vol. 85, no. 11, pp. 2917-2926, 2004.

[31] L. Ries and T. D. Sisk, "What is an edge species? The implications of sensitivity to habitat edges," Oikos, vol. 119, no. 10, pp. 1636-1642, 2010.

[32] S. P. Courtney and S. Courtney, "The "edge-effect" in butterfly oviposition: causality in Anthocharis cardamines and related species," Ecological Entomology, vol. 7, no. 2, pp. 131-137, 1982.

[33] L. C. Remer and S. B. Heard, "Local movement and edge effects on competition and coexistence in ephemeral-patch models," American Naturalist, vol. 152, no. 6, pp. 896-904, 1998.

[34] J. Olofsson, S. Stark, and L. Oksanen, "Reindeer influence on ecosystem processes in the tundra," Oikos, vol. 105, no. 2, pp. 386-396, 2004.

[35] J. M. Morales and S. P. Ellner, "Scaling up animal movements in heterogeneous landscapes: the importance of behavior," Ecology, vol. 83, no. 8, pp. 2240-2247, 2002.

[36] R. M. Ewers and R. K. Didham, "Pervasive impact of large-scale edge effects on a beetle community," Proceedings of the National Academy of Sciences of the United States of America, vol. 105, no. 14, pp. 5426-5429, 2008.

[37] L. Ries and T. D. Sisk, "Butterfly edge effects are predicted by a simple model in a complex landscape," Oecologia, vol. 156, no. 1, pp. 75-86, 2008.

[38] M. Holyoak, R. Casagrandi, R. Nathan, E. Revilla, and O. Spiegel, "Trends and missing parts in the study of movement ecology," Proceedings of the National Academy of Sciences of the United States of America, vol. 105, no. 49, pp. 19060-19065, 2008.

[39] A. M. Reynolds, "Animals that randomly reorient at cues left by correlated random walkers do the lévy walk," American Naturalist, vol. 175, no. 5, pp. 607-613, 2010.
[40] P. M. Kareiva, "Local movement in herbivorous insects: applying a passive diffusion model to mark-recapture field experiments," Oecologia, vol. 57, no. 3, pp. 322-327, 1983.

[41] R. Southwood and P. A. Henderson, Ecological Methods, Blackwell Science, Oxford, UK, 2000.

[42] E. M. Jakob, S. D. Marshall, and G. W. Uetz, "Estimating fitness: a comparison of body condition indices," Oikos, vol. 77, no. 1, pp. 61-67, 1996.

[43] P. Turchin, Quantitative Analysis of Movement: Measuring and Modeling Population Redistribution in Animals and Plants, Sinauer Associates, Sunderland, Mass, USA, 1998.

[44] J. F. Campbell and D. W. Hagstrum, "Patch exploitation by Tribolium castaneum: movement patterns, distribution, and oviposition," Journal of Stored Products Research, vol. 38, no. 1, pp. 55-68, 2002.

[45] C. Nansen, D. K. Weaver, S. E. Sing et al., "Within-field spatial distribution of Cephus cinctus (Hymenoptera: Cephidae) larvae in Montana wheat fields," Canadian Entomologist, vol. 137, no. 2, pp. 202-214, 2005.

[46] M. A. Villard, M. K. Trzcinski, and G. Merriam, "Fragmentation effects on forest birds: relative influence of woodland cover and configuration on landscape occupancy," Conservation Biology, vol. 13, no. 4, pp. 774-783, 1999.

[47] L. Imbeau, P. Drapeau, and M. Mönkkönen, "Are forest birds categorised as "edge species" strictly associated with edges?" Ecography, vol. 26, no. 4, pp. 514-520, 2003.

[48] L. Ries and D. M. Debinski, "Butterfly responses to habitat edges in the highly fragmented prairies of Central Iowa," Journal of Animal Ecology, vol. 70, no. 5, pp. 840-852, 2001.

[49] N. Schtickzelle and M. Baguette, "Behavioural responses to habitat patch boundaries restrict dispersal and generate emigration-patch area relationships in fragmented landscapes," Journal of Animal Ecology, vol. 72, no. 4, pp. 533-545, 2003.

[50] H. van Dyck and M. Baguette, "Dispersal behaviour in fragmented landscapes: routine or special movements?" Basic and Applied Ecology, vol. 6, no. 6, pp. 535-545, 2005.

[51] K. Dmowski and M. Kozakiewicz, "Influence of a shrub corridor on movements of passerine birds to a lake littoral zone," Landscape Ecology, vol. 4, no. 2-3, pp. 99-108, 1990.

[52] A. Desrochers and M. J. Fortin, "Understanding avian responses to forest boundaries: a case study with chickadee winter flocks," Oikos, vol. 91, no. 2, pp. 376-384, 2000.

[53] E. E. Barding and T. A. Nelson, "Raccoons use habitat edges in Northern Illinois," American Midland Naturalist, vol. 159, no. 2, pp. 394-402, 2008.

[54] E. E. Crone and C. B. Schultz, "Old models explain new observations of butterfly movement at patch edges," Ecology, vol. 89, no. 7, pp. 2061-2067, 2008.

[55] J. Clobert, J. F. Le Galliard, J. Côté, S. Meylan, and M. Massot, "Informed dispersal, heterogeneity in animal dispersal syndromes and the dynamics of spatially structured populations," Ecology Letters, vol. 12, no. 3, pp. 197-209, 2009. 

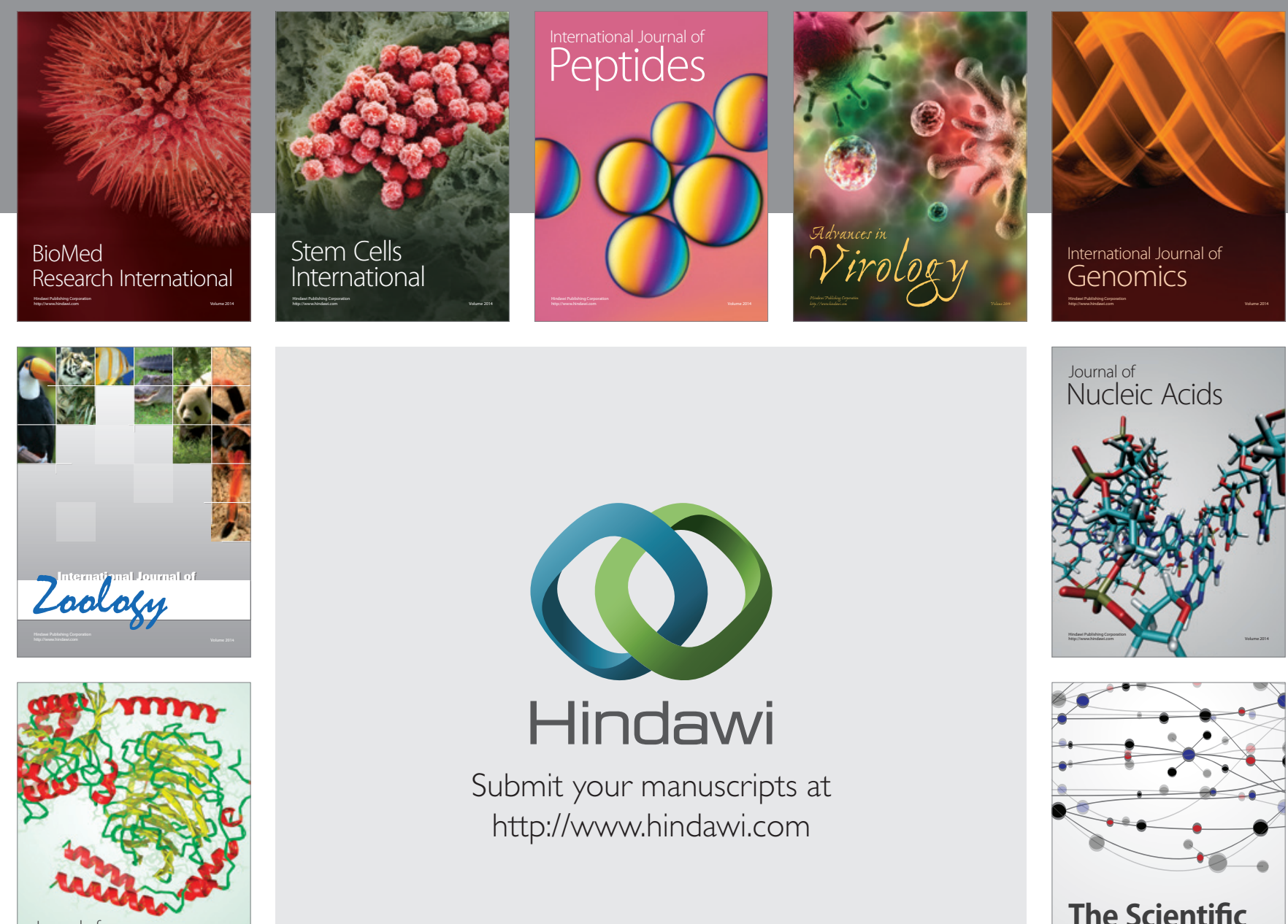

Submit your manuscripts at

http://www.hindawi.com

Journal of
Signal Transduction
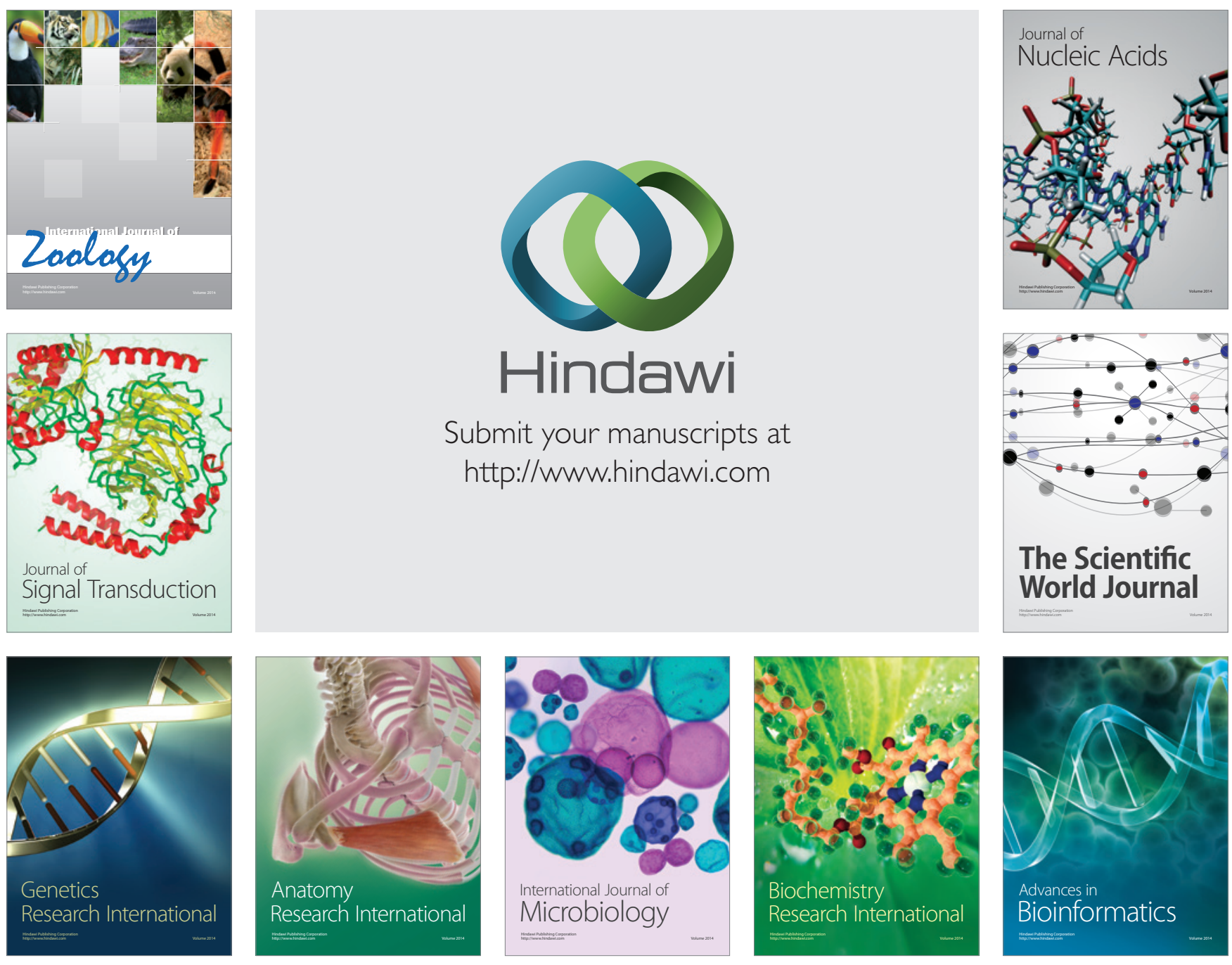

The Scientific World Journal
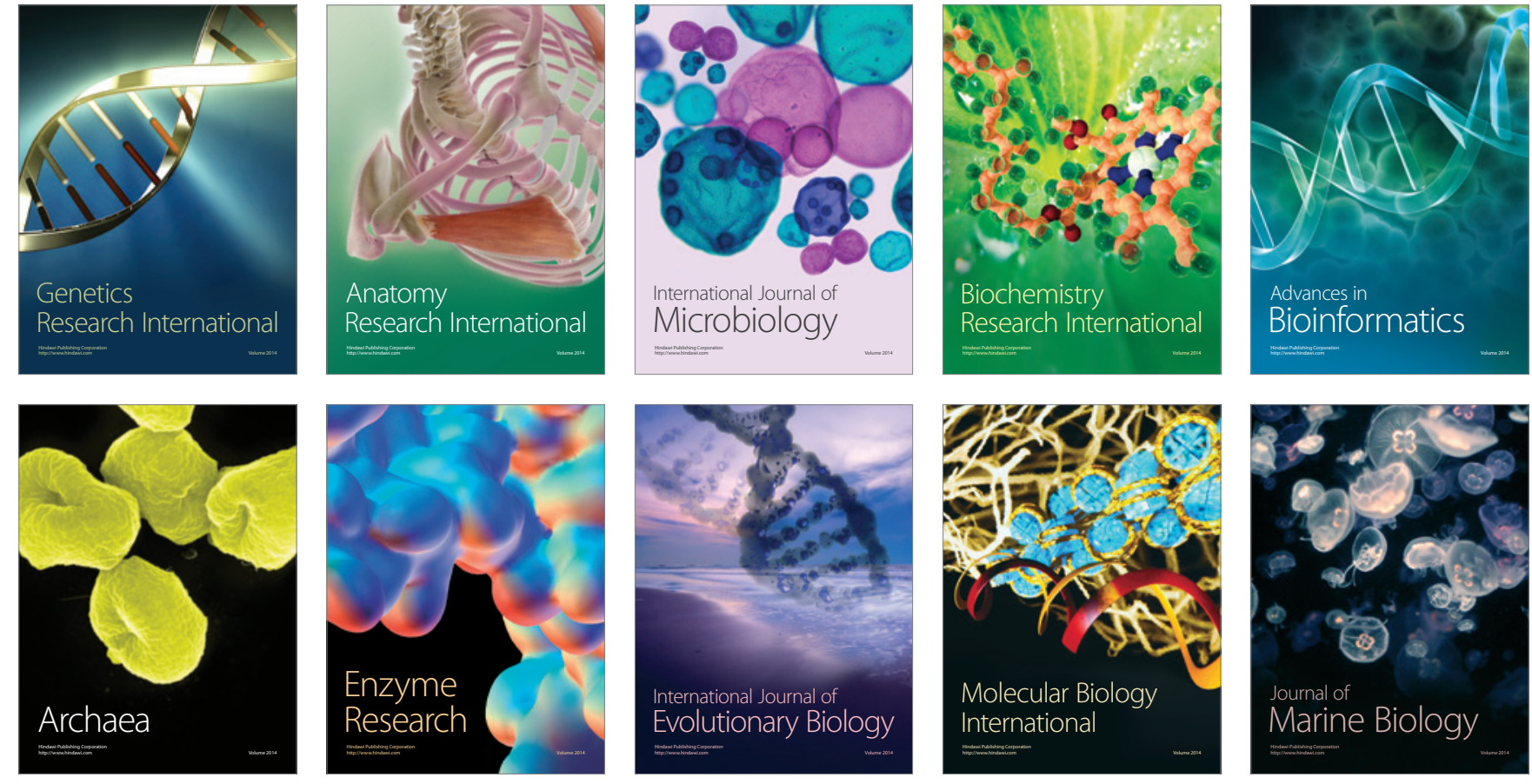